\title{
Korrekter Umgang mit Rabatten
}

Bruno Henggi ${ }^{a}$, Charlotte Schweizer ${ }^{b}$

${ }^{a}$ Verantwortlicher Public Affairs der FMH; ${ }^{b}$ Leiterin Abteilung Kommunikation der FMH

\section{Am 1.1.2020 treten neue Vorschriften betreffend den Umgang mit Heilmitteln in} Kraft. Die neuen Bestimmungen bringen strengere Regelungen mit sich, welche direkt für den Berufsalltag von Ärztinnen und Ärzten relevant sind. Sie sind in der Verordnung «Integrität und Transparenz im Heilmittelbereich (VITH)» festgehalten. Diese hat zum Ziel, die Integrität im Umgang mit Arzneimitteln zu stärken und dessen Transparenz zu erhöhen. Daher reguliert sie Preisrabatte und Abgeltungen seitens Arzneimittellieferanten stark und detailliert. Weiter enthält sie Bestimmungen über Unterstützungsbeiträge im Bereich Forschung sowie Weiterund Fortbildung. Auch für die Annahme von Geschenken oder Einladungen zum Essen gibt es strikte Vorschriften.

Für Ärztinnen und Ärzte bedeutet dies, dass sie sich mit den Änderungen der neuen Verordnung vertraut machen müssen, um die neuen Bestimmungen achtsam und sorgfältig umsetzen zu können. Weiter müssen Ärztinnen und Ärzte ihre diesbezüglichen Handlungen konsequent und umfassend dokumentieren. Als Berufsverband erachtet es die FMH als ihre Pflicht, ihre Mitglieder auf die strengere Regelung aufmerksam zu machen und sie kontinuierlich und regelmässig über die neuen gesetzlichen Bestimmungen zu informieren. Dies möchten wir in anschaulicher Form in einer Artikelserie in vier Teilen realisieren.

Im vorliegenden Beitrag gehen wir näher auf Fragen ein, welche sich im Zusammenhang mit der Gewährung von Rabatten stellen. Wir behandeln diese anhand von praktischen Beispielen, wie sie sich im Berufsalltag von Ärztinnen und Ärzten ergeben können.

\section{Lieferung zum Fabrikabgabepreis inklusive Transport}

Frage: Eine Ärztin erhält eine Lieferung an Arzneimitteln zum Fabrikabgabepreis. Der Transport wird nicht in Rechnung gestellt. Ist dies zulässig?

Antwort: Eine Lieferung von Arzneimitteln zum Fabrikabgabepreis ohne Inrechnungstellung der Transportkosten bedeutet bereits einen Preisrabatt. Um sich korrekt und regelkonform zu verhalten, müsste die Ärztin diese Vergünstigung (die Logistikkosten) bei Leistungen nach KVG auf jede einzelne Packung umrechnen und die Vergünstigung alsdann an die Patientinnen und Patienten weitergeben, das heisst, entsprechend weniger verrechnen und die weitergegebene Vergünstigung dokumentieren. Diese Lösung ist für einzelne Ärzte impraktikabel, weil sie mit einem grossen administrativen Mehraufwand verbunden ist. Die andere Möglichkeit wäre, dass die Ärztin dem Arzneimittellieferanten den Transport der Arzneimittel dennoch bezahlt. Für diesen Fall sollte dem Arzneimittellieferanten mitgeteilt werden, dass die Logistikkosten auf der Rechnung ausgewiesen werden müssen.

\section{Generelle Rabatte}

Frage: Ein Arzt erhält bei der Bestellung vom Arzneimittellieferanten einen generellen Rabatt von 5\% auf den Fabrikabgabepreis gewährt. Wie soll der Arzt damit umgehen?

Antwort: Bei diesem Rabatt handelt es sich mit grosser Wahrscheinlichkeit um eine weitergabepflichtige Vergünstigung, da der Rabatt nicht auf einer Gegenleistung des Arztes beruht. Diese Vergünstigungen (Rabatte) darf er nicht zurückbehalten, d.h., er muss sie an die Krankenversicherung oder an den Patienten weitergeben. Auch in diesem Fall gilt die Antwort zur obenstehenden Frage betreffend «Lieferung zum Fabrikabgabepreis inklusive Transport». Zusätzlich muss hier bei der Verrechnung von Arzneimitteln der Spezialitätenliste aber der Rabatt in der Höhe von $5 \%$ des Fabrikabgabepreises vom Publikumspreis in Abzug gebracht werden. 


\section{Naturalrabatt}

Frage: Angenommen, eine Fachperson erhält Naturalrabatte. Wie soll sie damit umgehen? Sind Naturalrabatte wie Musterpackungen zu behandeln? Ist die Gratisweitergabe zu dokumentieren?

Antwort: Unzulässig ist es, wenn ein Pharmaunternehmen mehr Arzneimittel liefert, als bestellt wurden. Solche zusätzlich gelieferten Arzneimittel sollten dem Pharmaunternehmen zurückgesendet werden, denn diese können nicht als Musterpackungen gelten. Für den Erhalt von Musterpackungen wird vorausgesetzt, dass die Initiative für die Zustellung von der Fachperson ausgeht. Da Musterpackungen nicht verkauft werden dürfen, wäre es vorteilhaft, eine Gratisweitergabe zu dokumentieren.

\section{Gesetzliche Grundlage VITH: Erläuterung}

Der Zweck der neuen gesetzlichen Grundlage ist es zu vermeiden, dass die Verschreibung, Abgabe, Anwendung oder der Einkauf von verschreibungspflichtigen Arzneimitteln durch Zuwendungen jedweder Art beeinflusst wird. Anstatt ein vollständiges Verbot von Zuwendungen durchzusetzen, definiert die neue Verordnung einen Ausnahmekatalog, der abschliessend auflistet, was rechtlich weiterhin möglich sein soll.

Unter diesen Ausnahmekatalog fallen auch beim Heilmitteleinkauf gewährte Preisrabatte oder Rückvergütungen, sofern sie keinen Einfluss auf die Wahl der Behandlung haben. Das zentrale Merkmal eines Preisrabatts besteht darin, dass dieser voraussetzungslos, d.h. ohne Gegenleistung der Fachperson, gewährt wird. Das Krankenversicherungsrecht verwendet für den gleichen Sachverhalt den Begriff Vergünstigung. Vergünstigungen sind Preisnachlässe, die ohne Gegenleistung der Fachperson gewährt werden. Somit handelt es sich beim krankenversicherungsrechtlichen Begriff der Vergünstigung um heilmittelrechtliche Preisrabatte. Vergünstigungen unterliegen der krankenversicherungsrechtlichen Weitergabepflicht. Das heisst, dass sie mittels Preisreduktion auf dem Publikumspreis an die Krankenversicherung oder an den Patienten weitergegeben werden müssen.

Bei Arzneimitteln der Spezialitätenliste existieren zwei vom BAG festgelegte Preise. Zum einen der Fabrikabgabepreis (ex factory-Preis) und zum anderen der Publikumspreis. Der Fabrikabgabepreis ist derjenige Preis, den das Pharmaunternehmen für das Arzneimittel «ab Rampe» verlangen darf. Der Publikumspreis ist derjenige Preis, den eine Fachperson dem Patienten oder der Krankenversicherung höchstens in Rechnung stellen darf. Nach der Verordnung ist jeder auf dem
Fabrikabgabepreis gewährte Preisnachlass ein Preisrabatt. Da der Fabrikabgabepreis keine Logistikkosten enthält, stellt die Lieferung von Arzneimitteln inklusive Transport zum Fabrikabgabepreis oder geringfügig darunter ohne Inrechnungstellung des Transports ebenfalls einen Preisrabatt dar. Die Preise der Spezialitätenliste können unter <http://www. spezialitaetenliste.ch/> geprüft werden, weshalb schnell herausgefunden werden kann, ob ein Preisrabatt vorliegt oder nicht.

Anders ist es bei Arzneimitteln, die nicht auf der Spezialitätenliste aufgeführt sind. Bei solchen ist immer dann ein Preisrabatt gegeben, wenn eine Differenz zwischen dem Standardpreis (üblicher Verkaufspreis) eines Produkts und dem im Rahmen des Kaufs effektiv bezahlten Preis besteht. Wenn der Lieferant keinen Rabatt auf dem üblichen Verkaufspreis bewirbt oder auf dem Bestellformular auszeichnet, ist es unter Umständen schwierig oder unmöglich, einen Preisrabatt überhaupt zu erkennen.

\section{Vereinbarung zu Rabatten}

Das Heilmittelrecht regelt, ob eine Fachperson überhaupt einen Vorteil von einem Pharmaunternehmen annehmen darf. Unter Vorteilen werden dabei zum Beispiel Preisrabatte und Rückvergütungen, Abgeltungen für Gegenleistungen (wie beispielsweise Mengenrabatte) sowie Unterstützungsbeiträge für die Forschung, Lehre, Infrastruktur oder Weiter- und Fortbildung verstanden. Das Krankenversicherungsrecht regelt demgegenüber, ob ein allfälliger Vorteil, also eine Vergünstigung, an die Krankenversicherung oder den Patienten weitergegeben werden muss.

Frage: Gibt es Vereinbarungen, welche ermöglichen, dass Fachpersonen generelle Rabatte ohne Gegenleistung behalten können?

Antwort: Die Verordnung sieht grundsätzlich die Möglichkeit vor, dass Vergünstigungen, d.h. Preisrabatte ohne Gegenleistung, teilweise von den Fachpersonen zurückbehalten werden können. Voraussetzung ist aber erstens die mehrheitliche Weitergabe der Vergünstigungen an die Patientinnen und Patienten oder die Krankenversicherung. Zweitens muss der nicht weitergegebene Anteil der Vergünstigung für die Verbesserung der Behandlungsqualität eingesetzt werden. Die FMH ist derzeit daran, für die Mitglieder eine Rahmenvereinbarung auszuhandeln, welche die Mindestvoraussetzungen beziehungsweise die verschiedenen gesetzlichen Auflagen für solche Vereinbarungen zwischen Leistungserbringern und Krankenversicherern erfüllt. Es stellt sich hier aber die Frage, ab welcher 
Grösse und ab welchem Organisationsgrad die Teilnahme für Arztpraxen sinnvoll sein kann.

\section{Gesetzliche Grundlage VITH: Erläuterung}

Versicherer und Leistungserbringer haben neu die Möglichkeit zu vereinbaren, dass die gewährten Vergünstigungen für Arzneimittel sowie Mittel und Gegenstände nicht vollumfänglich weitergegeben werden. Schliessen Versicherung und Leistungserbringer eine entsprechende Vereinbarung ab, so müssen sie beachten, dass mehr als fünfzig Prozent der gewährten Vergünstigung tatsächlich an den Schuldner der Vergütung weitergegeben werden. Die Vereinbarung muss schriftlich erfolgen. Der nicht weitergegebene Anteil der Vergünstigung muss nachweislich zur Verbesserung der Qualität der Behandlung eingesetzt werden. Die Parteien müssen in den Vereinbarungen darlegen, wie und zu welchem Zeitpunkt sie aufzeigen, inwiefern der Einsatz der einbehaltenen Vergünstigungen zu einer messbaren Verbesserung der Qualität der Behandlung geführt hat.

\section{Transparenz}

Frage: Eine medizinische Fachperson hat zulässige Preisrabatte beziehungsweise Rückvergütungen erhalten. Muss sie diese dokumentieren und schriftlich festhalten?

Antwort: Ja, das Heilmittelgesetz sieht vor, dass auch die einkaufende Person Preisrabatte und Rückvergütungen in den Geschäftsbüchern, Belegen und Rechnungen ausweisen und auf Anfrage dem BAG offenlegen muss. Die Einhaltung der Transparenzbestimmungen ist wichtig, denn andernfalls besteht die Gefahr einer Strafandrohung.

\section{Gesetzliche Grundlage VITH: Erläuterung}

Beim Heilmitteleinkauf gewährte Preisrabatte und Rückvergütungen sollen sowohl in den Rechnungen und Geschäftsbüchern der Einkäufer - also zum Beispiel der Ärztinnen und Ärzte - als auch auf Seiten der Hersteller oder Lieferanten nachvollziehbar ausgewiesen werden. Auf Verlangen sind sie auch gegenüber dem Bundesamt für Gesundheit offenzulegen.

\section{Verordnung über die Integrität und Transparenz im Heilmittelbereich (VITH)}

\section{Art. 8 Rabatte}

1 Ein Preisrabatt entspricht der Differenz zwischen dem Standardpreis eines Produkts und dem im
Rahmen einer Transaktion effektiv bezahlten Preis. Für Arzneimittel der Spezialitätenliste liegt ein Preisrabatt insbesondere vor, wenn der effektiv bezahlte Preis unter dem Fabrikabgabepreis liegt.

2 Die Lieferung einer grösseren Menge, als bestellt und in Rechnung gestellt wird, ist unzulässig.

\section{Abschnitt: Transparenz}

Art. 10

1 Sämtliche Preisrabatte und Rückvergütungen, die beim Heilmitteleinkauf Personen oder Organisationen gewährt werden, die Heilmittel verschreiben, abgeben, anwenden oder zu diesem Zweck einkaufen, sind dem Bundesamt für Gesundheit auf Verlangen offenzulegen.

2 Die Transparenzpflicht nach Artikel 56 HMG gilt nicht für den Einkauf von frei-verkäuflichen Arzneimitteln (Abgabekategorie E) und klassischen Medizinprodukten der Klasse I gemäss Anhang IX der Richtlinie 93/42/EWG4 über Medizinprodukte.

\section{Verordnung vom 27. Juni 1995 über die Krankenversicherung}

\section{Art. 76a Weitergabe der Vergünstigung}

1 Die Vergünstigung nach Artikel 56 Absatz 3 des Gesetzes ist durch den Leistungserbringer in der Rechnung nach Artikel 42 des Gesetzes aufzuführen und dem Schuldner der Vergütung weiterzugeben.

2 Fliessen die Vergünstigungen über niedrigere Kosten bereits in die Berechnung der Tarife und Preise der entsprechenden Leistung ein, so müssen diese nicht mehr im Rahmen der Rechnungsstellung separat ausgewiesen werden.

Art. 76b Vereinbarung über die nicht vollumfängliche Weitergabe von Vergünstigungen

1 Vereinbarungen nach Artikel 56 Absatz 3bis des Gesetzes werden in erster Linie zwischen den Verbänden der Leistungserbringer und der Versicherer abgeschlossen.

2 Die Vereinbarungen über die nicht vollumfängliche Weitergabe der Vergünstigung nach Artikel 56 Absatz 3bis des Gesetzes müssen schriftlich abgeschlossen werden und namentlich folgende Angaben enthalten:

a. Art und Umfang der Vergünstigung sowie Modalitäten zur transparenten Dokumentation in den Belegen und Rechnungen;

b. Verwendungszweck der nicht weitergegebenen Vergünstigung, einschliesslich des Ziels zur Verbesserung der Behandlungsqualität; 
c. Modalitäten des Nachweises der Verbesserung der Behandlungsqualität.

3 Die nicht weitergegebenen Mittel werden in erster Linie zugunsten national ausgerichteter Programme zur Verbesserung der Behandlungsqualität eingesetzt.

4 Die Versicherer und Leistungserbringer müssen das BAG über abgeschlossene Vereinbarungen unverzüglich informieren.

\section{Art. 76c Berichterstattung an das BAG}

1 Die Versicherer erstatten dem BAG Bericht über die Einhaltung der Vereinbarung nach Artikel 76b. Sie reichen den Bericht unverzüglich nach Beendigung der Vereinbarung dem BAG ein. Bei mehrjährigen Projekten reichen sie jährlich Zwischenberichte ein.

2 Jeder Bericht sowie jeder Zwischenbericht enthält mindestens folgende Angaben:

a. Nachweis des Einsatzes der nicht weitergegebenen Vergünstigungen zur Verbesserung der Behandlungsqualität; b. Evaluation der durch die Vereinbarung erreichten Verbesserungen gegenüber der ursprünglichen Behandlungsqualität.

3 Die Evaluation muss durch eine unabhängige Organisation unter Anwendung von wissenschaftlichen Methoden nach anerkannten Standards oder Leitlinien durchgeführt werden.

Wir empfehlen Ärztinnen und Ärzten Vorsichtsmassnahmen zu treffen. Namentlich empfiehlt es sich:

Zu klären, ob bei Arzneimittellieferungen dieTransportkosten als Preisrabatt erlassen wurden. Wenn dies der Fall ist, ist dafür zu sorgen, dass der Lieferant der Fachperson die Transportkosten in Rechnung stellt.

Zu prüfen, ob im Fall eines Rabatts beziehungsweise einer Vergünstigung eine Gegenleistung vorliegt. Wenn dies nicht der Fall ist, besteht bei Leistungen nach KVG eine Weitergabepflicht.

Sicherzustellen, dass zusätzlich gelieferte Arzneimittel an den Lieferanten zurückgeschickt werden.

Zu gewährleisten, dass Preisrabatte und Rückvergütungen in den Geschäftsbüchern ausgewiesen werden.

\section{\{FMH}

\section{Jetzt Projekt einreichen - bis 09.12.2019}

\section{Zukunftsweisende Qualitätsarbeit im Gesund- heitswesen gehört aufs Podest: Gewinnen Sie die Innovation Qualité, den Preis für Qualität in der Medizin! Melden Sie Ihr Projekt an auf www.innovationqualite.ch.}

